\title{
Properties of Sulfolane Based Aprotic Electrolytes
}

\author{
Josef Maca, ${ }^{*}$ Martin Frk, Zdenka Rozsivalova, Marie Sedlarikova \\ Department of Electrical and Electronic Technology, Brno University of Technology, \\ 616 00, Czech Republic
}

Received 26 July 2013; accepted 23 December 2013

\begin{abstract}
The article deals with description of rheological and electrical properties of solvents for electrolytes of lithium-ion batteries. Solvents mixture of dimethyl sulfone and sulfolane at different volume ratios and with a lithium salt $\left(\mathrm{LiClO}_{4}\right)$ appears as a potentially suitable electrolyte. In this work, we investigate the influence of different solvents and their mixtures in order to find a solvent which increases the fire safety of such battery. The aim of this experiment is to investigate the rheological properties, particularly density and dynamic viscosity of solvents with lithium salt in temperature dependence and to find the optimal composition of the electrolyte from the perspective of achieving the lowest dynamic viscosity and better electrical conductivity, because both quantities are closely related with Walden's rule. The vibration method is used to determine the values of dynamic viscosity.
\end{abstract}

Keywords: electrolytes, solvents, viscosity, density.

\section{Introduction}

Lithium-ion accumulators are among the power sources suitable especially for the mobile applications, medical devices and electric vehicles. Their advantage is high capacity to weight ratio. Because lithium is used, it is not possible to use water as solvent even though it's cheap, available, nontoxic and it possess appropriate rheological properties [1,2]. For lithium - ion batteries, aprotic liquid solvents or gels are used. Gel is in actual fact an electrolyte bounded in appropriate polymer $[3,4]$. Rheological properties of the electrolytes are of the most important non-electrical physical quantities. Knowledge of these properties, in particular the value of dynamic viscosity, is crucial for selecting the suitable electrolyte solvents usable in lithium-ion accumulators. Solvents mixture of

\footnotetext{
* Corresponding author. E-mail address: xmacaj00@stud.feec.vutbr.cz
} 
dimethylsulfone and sulfolane with a lithium salt (in particular case $\mathrm{LiClO}_{4}$ ) appears as a potentially suitable electrolyte [3,5]. The resulting ratio of the components depends on the desired properties to be achieved. In modern $\mathrm{Li}-$ ion batteries, organic solvents are used as part of the electrolyte, which are flammable. There are two main ways of battery safety increasing. The first one is the electronic protection. This is in practice performed by adding an integrated circuit which evaluated the temperature and controls charging and discharging current, voltage on the cell and in case of excess one of the criteria disconnects the damaged cell [6]. This action stops the positive heat feedback and prevents the whole battery from destroying. The second way is to use new less flammable or inflammable materials which can work in higher temperatures without destruction.

\section{Solvents}

Solvents are substances that allow dissolving another substance in it without a chemical reaction with the solvent. The dissolved substance can be removed again from the solvent without a chemical change of the solvent composition. Water is the most used solvent which has been paid a great attention in all the fields dealing with this issue. Inorganic solvents have been regarded as typical aprotic solvents for a long time. In few past decades was a minor attention given to the organic aprotic solvents. A lot of solvents, especially dipole aprotic solvents have appeared in new publications in most cases in connection with electrolytes and ions transfer. Correct selection of solvent for individual applications depends on several factors among which the physical properties are the most important. The solvent should be liquid at temperature and pressure in which it is used. We have to take into consideration the dynamic properties such as viscosity, diffusion coefficient and temperature conductivity. Electrical, optical and magnetic properties such as dipole moment, relative permittivity, refractive index, magnetic and electrical conductivity have impact on solvents behaviour. Even the molecular characteristics such as size and orientation of particles and relaxation time have their effect on dissolve ability [5,7]. Between solvents, requirements for batteries application include sufficient temperature range for liquid state especially in low temperatures, low vapour pressure at the maximum work temperature for given application to prevent the explosion, high relative permittivity, low viscosity and low molar mass for ensuring high ion mobility, chemical stability to the electrode material, availability, low cost, easy to clean and nontoxicity. The required solvents properties for use in electrolytes can be divided into two main groups: electrical and physical.

\section{Used solvents}

The sulfolane molecule is composed of a ring containing four carbon atoms and one sulphur atom. To the sulphur atom, two oxygen atoms are connected with double bond. The sulphur-oxygen bond is polar, which enables a good dissolving in water (it is completely miscible with water). The carbon ring is non-polar so it is possible to blend it with water and hydrocarbons. Sulfolane is used as an 
industry solvent and for purification of natural gas and the most used solvent for aromatics purification [3,5].

Dimethyl sulfone aka. methylsulfonylmethane (MSM), is an organic sulphur compound. At room conditions, it is a white crystalline solid. Dimethyl sulfone is used in high temperature industry such as in organic and inorganic chemistry where it is used as extraction and reaction solvent, metal and fungicide treatment. Selected properties of used solvents are shown in Table 1.

Table 1. Selected properties of used solvents.

\begin{tabular}{ccccc}
\hline Solvent & Density $\left(\mathrm{kg} \mathrm{m}^{3}\right)$ & Viscosity $(\mathrm{mPa}$ s) & Permittivity $(-)$ & Melting point $\left({ }^{\circ} \mathrm{C}\right)$ \\
\hline Dimethyl sulfone & 1450 & $1.14\left(\right.$ at $\left.123^{\circ} \mathrm{C}\right)$ & 48 & 110.0 \\
Sulfolane & 1261 & $10.07\left(\right.$ at $\left.30^{\circ} \mathrm{C}\right)$ & 44 & 27.5 \\
\hline
\end{tabular}

\section{Physical quantities and measurement method Dynamic viscosity}

Viscosity (dynamic or kinematic) is a characteristic property of liquids and it expresses the rate of internal fluid friction that occurs at mutual motion of adjacent layers parallel to each other. It is closely linked to the internal structure of liquids. The course of viscosity varies considerably with temperature. The temperature dependence has always decreasing character which is in most cases possible to express by the empirical Arrhenius mathematical function in the form

$$
\eta=A \cdot e^{\frac{b}{T}}
$$

where $A$ is a characteristic material constant dependent on the frequency of thermal vibration and on distance of individual liquid molecules (sometimes referred to as the viscosity $\eta_{\infty}$ at high temperatures, $\left.T \rightarrow \infty\right)$ and physical quantity $b$ equals to $b=E_{A} / R$ ( $E_{A}$ is the flow activation energy and $R$ is the universal gas constant). This relation is also often used to represent experimental viscosity data for pure fluid and fluid mixtures solutions $[8,9,10]$. The vibration method was used to determine the values of dynamic viscosity. The general principle of this viscosity measurement method consists in the transfer of current on the apparent viscosity. The value of current is proportional to keeping constant frequency and amplitude of the vibrations of the sensor plates immersed in the observed liquid, on the apparent viscosity. Viscometer A\&D SV series is composed of two thin sensor plates with gold surface treatment that are driven with electromagnetic force at the same frequency by vibrating at constant sine-wave vibration in reverse phase like a tuning-fork. The electromagnetic driver controls the vibration of the sensor plates to keep in constant amplitude (see Fig. 1a). The driving electric current, which is related to exciting force, will be detected as the magnitude of viscosity produced between the sensor plates and the sample fluid. The coefficient of viscosity is obtained by the correlation between the driving electric current and the magnitude of viscosity [11]. Conversion of apparent viscosity $\eta_{a p}$ on dynamic $\eta$ is conditioned by knowledge of the sample density. 
a)

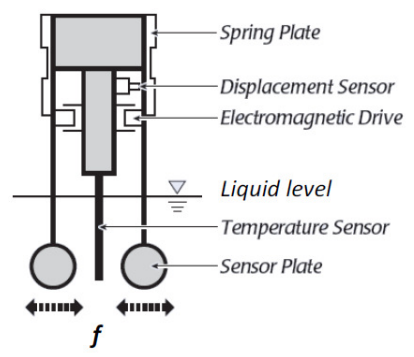

b)

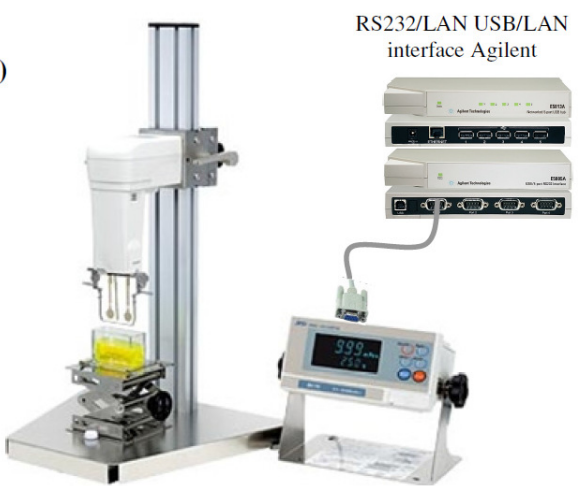

Figure 1. a) Scheme and physical principle of the vibration viscometer A\&D [11]; b) connection of the viscometer to the Internet.

\section{Density}

An analytical balance Ohaus Explorer EX223 with an extension kit for measuring the density of solid and liquid materials (see Fig. 2a), based on the principle of Archimedes law was used to determine the density of each solution [12]. The density determination kit includes a calibrated sinker (see Fig. 2b) of defined volume (in this case $V=10.000 \pm 0.005 \mathrm{~cm}^{3}$ ), whose mass is set in the air $\left(m_{1}\right)$ and subsequently in the observed solution $\left(m_{2}\right)$ with the desired temperature.
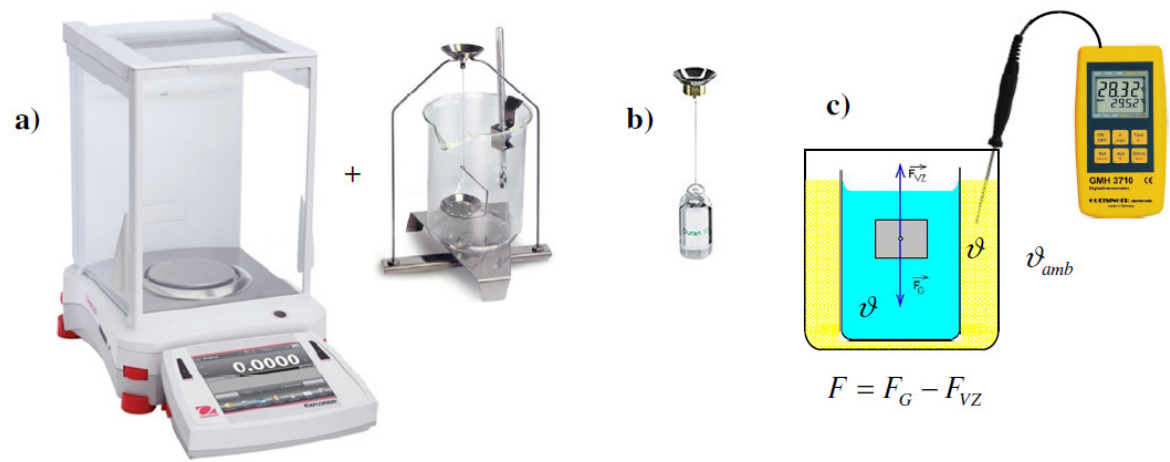

Figure 2. a) Instrumentation Ohaus including density determination kit; b) detail of glass sinker; c) the physical principle and the thermal stabilization of the solution in the course of the experiment.

The formula for calculating the density of a liquid $\rho_{\text {liq }}$ can be derived from the resultant force $F$ acting on the immersed sinker (see Fig. 2c) in the liquid and takes the form

$$
\rho_{\text {liq }}=\frac{m_{1}-m_{2}}{V}+\rho_{\text {air }}
$$

that respects the measured data, including the effects of air density $(0.0012$ $\left.\mathrm{g} \cdot \mathrm{cm}^{-3}\right)$.

\section{Specific conductivity}

The specific conductivity was determined by impedance spectroscopy measurement at ambient conditions. The values of equivalent circuit elements were determined from the obtained Nyquist graph. The equivalent circuit was 
R1+Q1. From the resulting Nyquist graph, the most linear part at low frequencies was selected and fitted on the chosen equivalent circuit. The calculation of impedance and all elements were done by help of the following equation

$$
Z(f)=R_{1}+\frac{1}{Q_{1}(j 2 \pi f)^{\alpha_{1}}}
$$

where $R_{1}$ is the electrolytes resistivity, $Q_{1}$ is the constant phase source, $f$ is the frequency. The impedance spectroscopy was made in the frequency range from 1 $\mathrm{MHz}$ to $100 \mathrm{~Hz}$. Two platinum electrodes conductivity cell covered by platinum black was used for measurement. All samples have the same concentration of dissolved salt $\left(1 \mathrm{~mol} \cdot \mathrm{L}^{-1}\right.$ of $\left.\mathrm{LiClO}_{4}\right)$.

\section{Sample preparing}

The samples of electrolyte were prepared with different concentration ratios of solvents as sulfolane and dimethyl sulfone (both from Sigma-Aldrich Corporation). First the saturation level of dimethyl sulfone in the second solvent was assessed and then the samples with volume concentration of dimethyl sulfone from $5 \%$ to $30 \%$ in five percent step were prepared. The samples were heated on temperature slightly above the melting temperature of dimethyl sulfone and after ideal mixing they have been let to cool down to room condition. After the cooling, recrystallized dimethyl sulfone was visually detected in the samples. The interval between two samples in which the dimethyl sulfone has and hasn't recrystallized was spitted more precisely, in our case in $1 \%$ steps. The sample testing was repeated and the saturation border of dimethyl sulfone in second solvent was determined. The saturation border was determined on $17 \%$ dimethyl sulofone in sulfolan. The amount of dimethyl sulfone in sulfolane varied from $0 \%$ volume percent to the saturation of dimethyl sulfone at $17 \%$ volume percent. The lithium salt $\mathrm{LiClO}_{4}$ (1 M concentration) was added and dissolved into all the samples. Into the beaker with sulfolane heated to $40{ }^{\circ} \mathrm{C}$ was added dimethyl sulfone (under the room conditions solid state). The weight of dimethyl sulfone was calculated from knowledge of the density values and the required volume. After melting and mutual mixing of solution components, a defined amount of lithium salt $\left(\mathrm{LiClO}_{4}\right.$ - lithium perchlorate) was added into the mixture. The resulting sample of solution was blended in a closed vessel on magnetic stirrer for another 24 hours.

\section{Experiment}

\section{Density determination of solutions}

Densities of various concentrated solutions were monitored in the temperature range from 5 to $90{ }^{\circ} \mathrm{C}$ with a step of approximately $20^{\circ} \mathrm{C}$. From the obtained and approximated temperature dependences of density it is possible, among other things, to determine the coefficients of volume expansion of solutions which in liquid materials are not generally negligible. To achieve the lower and higher temperature than the ambient conditions, a climatic chamber CLIMACEL 111 
was used, in which the temperature stabilization of all the solutions samples was done. The beaker with the solution was immersed in the vessel with synthetic organic ester fluid to ensure sufficient thermal stability in the course of the density measurement. A digital thermometer GREISINGER 3710 with a probe Pt100 Class A was used to determine the actual temperature of the solutions (see Fig. 2c). The volumetric changes of the solution, respectively calculated density values in the temperature dependence were approximated by the mathematical function in the form,

$$
V=V_{20}(1+\beta \Delta \vartheta) \text { resp. } \rho=\frac{\rho_{20}}{(1+\beta \Delta \vartheta)}
$$

which takes into account only the linear coefficient of volume expansion $\beta$. The quadratic term which is very small is applied in the case of higher temperature differences $\Delta \vartheta$ and therefore is neglected here. Temperature dependencies of the density of the solutions with limit DMSO2 concentrations (0 and $17 \%$ volume percent) are shown in Fig. 3.

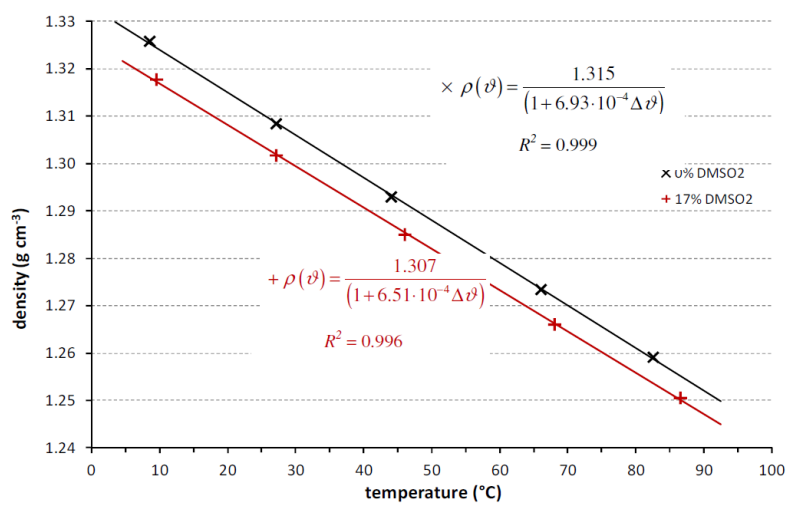

Figure 3. Temperature dependencies of the density of solutions.

The displayed curves create a sector. Within this area, the dependencies are situated for solutions with other concentrations, but for greater lucidity are not shown graphically. The calculated values of the coefficient of volume expansion $\beta$ and density of the solutions $\rho_{20}$ at $20{ }^{\circ} \mathrm{C}$ are listed in Table 2 with the volume percentage dimethyl sulfone (DMSO2) as a parameter.

Table 2. Selected physical properties of the investigated solutions at various concentrations.

\begin{tabular}{cccccccc}
\hline vol $\%$ DMSO2 & $0 \%$ & $3 \%$ & $6 \%$ & $9 \%$ & $12 \%$ & $15 \%$ & $17 \%$ \\
\hline$\rho_{20}\left(\mathrm{~g} . \mathrm{cm}^{-3}\right)$ & 1.315 & 1.315 & 1.313 & 1.310 & 1.310 & 1.309 & 1.307 \\
$\beta\left(10^{-4} \mathrm{~K}^{-1}\right)$ & 6.93 & 6.86 & 6.86 & 6.89 & 7.17 & 6.58 & 6.51 \\
\hline
\end{tabular}

\section{Dynamic viscosity measurement of solutions}

The automated laboratory workplace was assembled to measure rheological properties of the fluids in temperature range. Also, Agilent graphical language environment VEE Pro was used for the communication between computer and viscometer A\&D SV 10 or analytical balance Ohaus Explorer Ex223. Serial communication interfaces of viscometer and bath thermostat were connected to 
the USB/RS232 interface Agilent E5805A, whose USB output was directly interconnected to the Networked LAN/USB hub Agilent E5813A that provides access to the Internet (see Fig. 1b). This way, it is possible to remotely control the instruments which are equipped with serial interface RS232 only over the Internet. Before starting the experiment, the calibration of vibration viscometer A\&D SV-10 was performed. Regarding the nature of the experiment and the expected range of the measured viscosity values, two-point calibration was chosen. Certified Newtonian fluid "Fluid 50" in the form of silicone oil (provides excellent temperature stability and is less temperature sensitive than other viscosity standards; dynamic viscosity is equal $49.4 \mathrm{mPa} \cdot \mathrm{s}$ at temperature $25^{\circ} \mathrm{C}$ ) and pure distilled water (dynamic viscosity is equal $0.89 \mathrm{mPa} \cdot \mathrm{s}$ at temperature 25 ${ }^{\circ} \mathrm{C}$ ) were used as the viscosity standards. Calibrated interval of dynamic viscosity fully covers the range of measured values of the viscosity of sulfolane-based solvents with different volume percentage of dimethyl sulfone. The measured apparent viscosity values $\eta_{a p}$ were converted to dynamic viscosity for all the measured temperature according to the relationship

$$
\eta(T)=\frac{\eta_{a p}}{\rho_{r e l}(T)}
$$

where $\rho_{r e l}$ is the relative density of the sample, i.e., the value of the density related to the unit density to which the instrument is calibrated. Results are shown in Table 2.

Temperature dependencies of the dynamic viscosity of the solutions with limit DMSO2 concentrations ( 0 and $17 \%$ volume percent) are again shown in Fig. 4 (discreet points). As one can see from this figure, the viscosity of solutions monotonically decreases with increasing temperature. Temperature dependencies of viscosity were approximated by the Arrhenius function using the least squares method and the resultant courses are plotted graphically in Fig. 4 (solid line) including relevant equations.

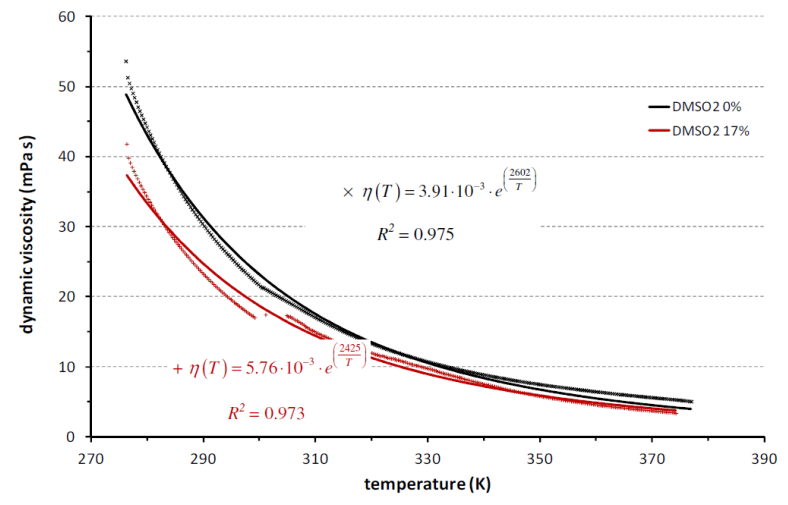

Figure 4. Temperature dependencies of the dynamic viscosity.

\section{Specific conductivity measurement of electrolytes}

The measurement was realized by a commercial conductivity cell KC 503 from company THETA '90. This glass body (two electrode cell with platinum circle electrodes) was immersed in a bottle with the sample. The specific conductivity (see Fig. 5) was determined from electrochemical impedance spectroscopy (EIS) 
using an automated potentiostat BioLogic at ambient temperature with the following setting:

Mode Single Sine

Frequency range: $1 \mathrm{MHz}$ to $100 \mathrm{~Hz}$;

Peak-to-peak AC voltage: $10 \mathrm{mV}$;

Average number of measurements for one frequency: 5;

Number of cycles: 10 .

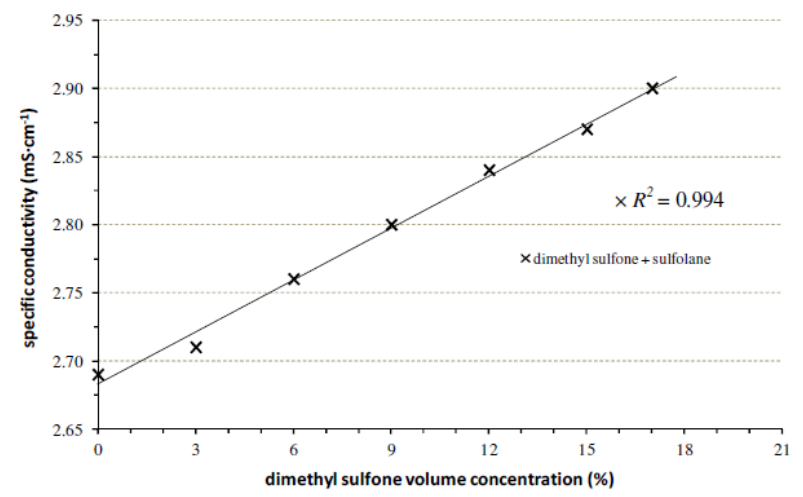

Figure 5. Specific conductivity on DMSO2 concentrations dependence.

\section{The Walden law and activation energy}

Logarithmic dependencies of viscosity and specific conductivity were used for determination of coefficient $\alpha$, in modified Walden law in the form

$$
\eta \cdot \sigma^{\alpha}=\text { const } \text {. }
$$

where $\alpha$ is a modified coefficient. The searched coefficient is then the slope of the linear dependence (Fig. 6).

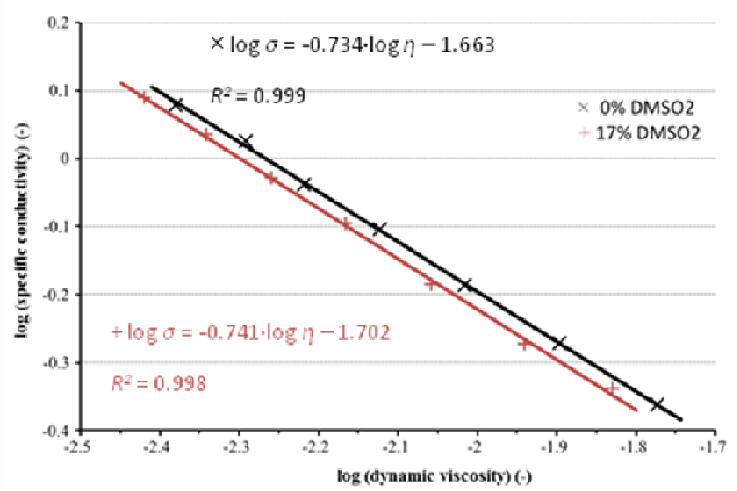

Figure 6. Modified Walden law representation for SL-DMSO2 system.

The apparent activation energy was determined from temperature dependencies of viscosity and specific conductivity in the temperature range 6 to $120{ }^{\circ} \mathrm{C}$ for viscosity, and 25 to $90{ }^{\circ} \mathrm{C}$ for specific conductivity. The apparent activation energy was calculated from Arrhenius equation (1). 


\section{Conclusions}

Theoretical information about vibration method of viscosity measurement was summarized and initial results were published in the article. Dynamic viscosity of seven solutions of dimethyl sulfone and sulfolane at different volume ratios $(0,3$, $6,9,12,15$ and 17 vol \% DMSO2) and with a lithium salt $\left(\mathrm{LiClO}_{4}\right)$ have been measured with vibration techniques in temperature range from 275 to $375 \mathrm{~K}$. All of these solutions have approximately the same value of the volume expansion coefficient which is of around $6.90 .10^{-4} \mathrm{~K}^{-1}$. Solution (17 vol \% DMSO2) exhibited the lowest value of the dynamic viscosity in whole temperature range and appears as potentially more suitable for electrolyte from the perspective of rheological properties. The values of flow activation energy will be calculated from the temperature dependencies of dynamic viscosity in the course of other experimental activities. Subsequently, the behaviour of solutions will be examined in DC and AC electric field to obtain the frequency dependencies of relative permittivity and electrical conductivity which is closely related to dynamic viscosity in accordance with Walden's rule. The modified Walden law was applied on the sulfolane - dimethyl sulfone system. The coefficient $\alpha$ varies between 0.734 - 0.741 for DMSO2 according to the concentration change (Fig. 6). The apparent activation energy for viscosity and conductivity was calculated: 0.243 for viscosity dependence and 0.161 for specific conductivity dependence. The difference between $E_{a}(\sigma)$ and $E_{a}(\eta)$ can be caused by solvated ion pairs which are electric neutral in the external electric field but they still have influence on the viscosity. Using a solvent with higher flash point (sulfolane) can also increase the work temperature of the battery and that allows us to use higher charge and discharge currents and that reduce the time needed to battery charging. Disadvantage of sulfolane is its high melting point $\left(27.5^{\circ} \mathrm{C}\right)$ and that decreases the temperature use window in which the battery can work reliably. The melting point can be reduced due to sulfolane's high cryoscopy constant $65.5 \mathrm{~K} \cdot \mathrm{kg} \cdot \mathrm{mol}^{-1}[13]$ by adding a salt which is important for electrolyte function, or even by blending a second solvent into sulfolane, which rapidly sinks the melting point under zero temperature [14]. The combination of sulfolane and dimethyl sulfone is suitable for the batteries which work always at elevated temperature, e.g., in the proximity of heating devices. Using appropriate combination of solvents makes it possible to reach sufficiently high specific conductivity for use in batteries. The specific conductivity has increased by addition of dimethyl sulfone.

\section{Acknowledgements}

This work was supported by the grant FEKT-S-11-7 "Materiály a technologie pro elektrotechniku" and project CZ.1.07/2.3.00/20.0103 "Support for human resources and transfer of knowledge in conditions of international cooperation of research teams“.

\section{References}

1. A\&D Company Limited. SV-A Series Users' Handbook Version 1.13E. 2009. P. 196. 
2. Horvath AL. Handbook of aqueous electrolyte solutions: physical properties, estimation methods and correlation methods. West Sussex: Ellis Horwood;1985. P. 631.

3. Watanabe Y, Kinoshita S, Wada S, et al. J Power Sources. 2008;179:770779.

4. Zhou YF, Xie S, Ge XW, et al. J Appl Electrochem. 2005;34:1119-1125.

5. $\quad$ Li S, Li B, Xu X, et al. J Power Sources. 2012;209:295-300.

6. Kocman V. Physics and technology of electrical materials Insulator A. Brno: SNTL;1971. P. 526.

7. Lance RC, Barnard Jr AJ, Hooyman JE. J Hazard Mater. 1979;3:107-119.

8. Reid RC, Prausnitz JM, Poling BE. The properties of gases and liquids. 4th ed. New York: McGaw-Hill;1987. P. 768.

9. Brouillette D, Perron G, Desnoyers J. Electrochim Acta. 1999;44:47214742.

10. Águila-Hernández J, Trejo A, García-Flores B, et al. Fluid Phase Equilibr. 2008;267:172-180.

11. Abouimrane A, Belharouak I, Amine K. Electrochem Comm. 2009;11:1073-1076.

12. Marcus Y. The properties of solvents. Chichester: John Wiley \& Sons Ltd;1998. P.399.

13. Monica MD, Jannelli L, Lamanna U. J Phys Chem. 1967;71:1068-1071.

14. Maca J, Sedlarikova M, Vondrak J, et al. Phase diagram for mixtures of sulfolane - dimethylcarbonate for using in electrolytes for lithium - ion batteries. Proceeding of the ABAF- 12 Meeting, 2011. P. 65-68. 